\title{
Non-Ohmic Coulomb drag in the ballistic electron transport regime
}

\author{
V. L. Gurevich and M. I. Muradov \\ Solid State Physics Department, A. F. Ioffe Institute, 194021 Saint Petersburg, Russia
}

\begin{abstract}
We work out a theory of the Coulomb drag current created under the ballistic transport regime in a one-dimensional nanowire by a ballistic non-Ohmic current in a nearby parallel nanowire. As in the Ohmic case, we predict sharp oscillation of the drag current as a function of gate voltage or the chemical potential of electrons. We also study dependence of the drag current on the voltage $V$ across the driving wire. For relatively large values of $V$ the drag current is proportional to $V^{2}$.
\end{abstract}

The purpose of the present paper is to study the Coulomb drag current in the course of ballistic (collisionless) electron transport in a nanowire due to a ballistic driving non-Ohmic current in an adjacent parallel nanowire. The possibility of the Coulomb drag effect in the ballistic regime in quantum wires has been demonstrated by Gurevich, Pevzner and Fenton [四] and has been experimentally observed by Debray et al. [2]. If two wires, 1 and 2, are near one another and are parallel, the drag force due to the ballistic current in wire 2 acts as a sort of permanent acceleration on the electrons of wire 1 via the Coulomb interaction.

We assume that the largest dimension of the structure is smaller than the electron mean free path in the problem (typically a few $\mu \mathrm{m}$ ). Such nanoscale systems are characterized by low electron densities, which may be varied by means of the gate voltage.

\footnotetext{
${ }^{1} \mathrm{~A}$ number of references to early papers on the Coulomb drag is given in [1].
} 
Following [1] we assume that the drag current in wire 1 is much smaller than the driving ballistic current in wire 2 and calculate the drag current by iterating the Boltzmann equation for wire 1 . We have

$$
v \frac{\partial F^{(1)}}{\partial z}=-I^{(12)}\left\{F^{(1)}, F^{(2)}\right\}
$$

where $F^{(1,2)}$ are the electron distribution functions in wires 1 and 2 respectively, $v=p / m$ is the electron velocity, $p$ is the $x$-component of the electron quasimomentum, the $x$ axis is parallel to the wires. The collision integral takes into account only the interwire electronelectron scattering, so that otherwise the electron motion in both wires is considered as ballistic. Now,

$$
I^{(12)}\left\{F^{(1)}, F^{(2)}\right\}=\sum_{n^{\prime} p^{\prime} q} W_{1 p n, 2 p^{\prime} n^{\prime}}^{1 p+q n, 2 p^{\prime}-q n^{\prime}} \mathcal{P}
$$

where

$$
\mathcal{P}=\left[F_{n p}^{(1)} F_{n^{\prime} p^{\prime}}^{(2)}\left(1-F_{n p+q}^{(1)}\right)\left(1-F_{n^{\prime} p^{\prime}-q}^{(2)}\right)-F_{n p+q}^{(1)} F_{n^{\prime} p^{\prime}-q}^{(2)}\left(1-F_{n p}^{(1)}\right)\left(1-F_{n^{\prime} p^{\prime}}^{(2)}\right)\right] .
$$

As in Ref. [1], we assume the wires to be different though having the same lengths $L$ and consider the interaction processes when electrons in the two nanowires after scattering remain within the initial subbands $\varepsilon_{n p}^{(1)}=\varepsilon_{n}^{(1)}(0)+p^{2} / 2 m$ and $\varepsilon_{n^{\prime} p}^{(2)}=\varepsilon_{n^{\prime}}^{(2)}(0)+p^{2} / 2 m, n$ being the bands' number.

The first iteration of Eq.(1) gives for the nonequilibrium part of the distribution function $\Delta F_{n p}^{(1)}$

$$
\Delta F_{n p}^{(1)}=-\left(z \pm \frac{L}{2}\right) \frac{1}{v_{n p}} I^{(12)}\left\{F^{(1)}, F^{(2)}\right\}
$$

for $p>0(p<0)$ respectively. Using the particle conserving property of the scattering integral

$$
\sum_{n} \int d p I^{(12)}\left\{F^{(1)}, F^{(2)}\right\}=0
$$

we get for the drag current 


$$
J=-2 e L \sum_{n} \int_{0}^{\infty} \frac{d p}{2 \pi \hbar} I^{(12)}\left\{F^{(1)}, F^{(2)}\right\} .
$$

The scattering probability $W_{1 p n, 2 p^{\prime} n^{\prime}}^{1 p+q, 2 p^{\prime}-q n^{\prime}}$ in Eq.(2) includes a delta-function describing the energy conservation for the electrons belonging to two different wires

$$
W_{1 p n, 2 p^{\prime} n^{\prime}}^{1 p+q n, 2 p^{\prime}-q n^{\prime}}=\frac{2 \pi}{\hbar}\left|V_{1 p n, 2 p^{\prime} n^{\prime}}^{1 p+q n, 2 p^{\prime}-q n^{\prime}}\right|^{2} \delta\left(\varepsilon_{n p}^{(1)}+\varepsilon_{n^{\prime} p^{\prime}}^{(2)}-\varepsilon_{n p+q}^{(1)}-\varepsilon_{n^{\prime} p^{\prime}-q}^{(2)}\right)
$$

which following Ref. [1] can be brought into the form

$$
\delta\left(\varepsilon_{n p}^{(1)}+\varepsilon_{n^{\prime} p^{\prime}}^{(2)}-\varepsilon_{n p+q}^{(1)}-\varepsilon_{n^{\prime} p^{\prime}-q}^{(2)}\right)=\frac{m}{\left|p-p^{\prime}\right|} \delta\left(q+p-p^{\prime}\right) .
$$

This means that here we have backscattering processes and the electrons swap their quasimomenta as a result of collision.

To calculate the drag current we do the first iteration of the Boltzmann equation in the collision term. One can insert the equilibrium distribution functions into the collision term, e.g. $F_{n p}^{(1)}=f\left(\varepsilon_{n p}^{(1)}-\mu\right)$ for the first wire. Here $f(\varepsilon-\mu)$ is the Fermi function.

We assume, in the spirit of the Landauer-Buttiker-Imry [3, [4] approach, the driving quantum wire to be connected to reservoirs which we call 'left' $(l)$ and 'right' $(r)$, each of them being in independent equilibrium described by shifted chemical potentials $\mu^{(l)}=\mu-e V / 2$ and $\mu^{(r)}=\mu+e V / 2$. Here $\mu$ is the average chemical potential while $\Delta \mu / e=V$ is the voltage across wire 2 (we will assume that $e V>0$ ) and $e<0$ is the electron charge. Therefore, the electrons entering the wire from the 'left' ('right') and having quasimomenta $p^{\prime}>0\left(p^{\prime}<0\right)$ are described by

$$
\begin{array}{ll}
F_{n^{\prime} p^{\prime}}^{(2)}=f\left(\varepsilon_{n^{\prime} p^{\prime}}^{(2)}-\mu^{(l)}\right) & p^{\prime}>0, \\
F_{n^{\prime} p^{\prime}}^{(2)}=f\left(\varepsilon_{n^{\prime} p^{\prime}}^{(2)}-\mu^{(r)}\right) & p^{\prime}<0
\end{array}
$$

and we see that the collision integral Eqs. (2), (3) is identically zero if the initial quasimomentum $p^{\prime}$ and the final quasimomentum $p^{\prime}-q$ of electron are of the same sign. In other words we have here backscattering processes; otherwise the equilibrium distribution functions on the right-hand side of Eqs. (22), (3) would depend on the same chemical potential and the collision term would vanish. 
Due to Eq.(8) we will be interested only in the values $p^{\prime}<0$ [because of the restriction $p^{\prime}-q=p>0$ which follows from Eq.(6)] and get the following product of distribution functions in the collision term Eqs. (2) and (3)

$$
\mathcal{P}=F_{n p}^{(1)} F_{n^{\prime} p^{\prime}}^{(2 r)}\left(1-F_{n p^{\prime}}^{(1)}\right)\left(1-F_{n^{\prime} p}^{(2 l)}\right)-F_{n p^{\prime}}^{(1)} F_{n^{\prime} p}^{(2 l)}\left(1-F_{n p}^{(1)}\right)\left(1-F_{n^{\prime} p^{\prime}}^{(2 r)}\right)
$$

or

$$
\begin{aligned}
\mathcal{P} & =f\left(\varepsilon_{n p}^{(1)}-\mu\right) f\left(\varepsilon_{n^{\prime} p^{\prime}}^{(2)}-\mu^{(r)}\right)\left[1-f\left(\varepsilon_{n p^{\prime}}^{(1)}-\mu\right)\right]\left[1-f\left(\varepsilon_{n^{\prime} p}^{(2)}-\mu^{(l)}\right)\right] \\
& -f\left(\varepsilon_{n p^{\prime}}^{(1)}-\mu\right) f\left(\varepsilon_{n^{\prime} p}^{(2)}-\mu^{(l)}\right)\left[1-f\left(\varepsilon_{n p}^{(1)}-\mu\right)\right]\left[1-f\left(\varepsilon_{n^{\prime} p^{\prime}}^{(2)}-\mu^{(r)}\right)\right] .
\end{aligned}
$$

This expression can be recast into the form

$$
\begin{aligned}
\mathcal{P} & =2 \sinh \left(e V / 2 k_{\mathrm{B}} T\right) \exp \left\{\left(\varepsilon_{n p}^{(1)}-\mu\right) / k_{\mathrm{B}} T\right\} \exp \left\{\left(\varepsilon_{n \prime p \prime}^{(2)}-\mu\right) / k_{\mathrm{B}} T\right\} \\
& \times f\left(\varepsilon_{n p}^{(1)}-\mu\right) f\left(\varepsilon_{n^{\prime} p^{\prime}}^{(2)}-\mu-e V / 2\right) f\left(\varepsilon_{n p^{\prime}}^{(1)}-\mu\right) f\left(\varepsilon_{n^{\prime} p}^{(2)}-\mu+e V / 2\right)
\end{aligned}
$$

and for the drag current we have

$$
J=-2 e \sinh \left(\frac{e V}{2 k_{\mathrm{B}} T}\right) \frac{2 \pi}{\hbar} \frac{m L}{2 \pi \hbar}\left(\frac{2 L}{2 \pi \hbar}\right)^{2}\left(\frac{2 e^{2}}{\kappa L}\right)^{2} \sum_{n n^{\prime}} \int_{0}^{\infty} d p \int_{0}^{\infty} d p^{\prime} \frac{g_{n n^{\prime}}\left(p+p^{\prime}\right)}{p+p^{\prime}} \mathcal{Q}
$$

where

$$
\mathcal{Q}=\exp \frac{\varepsilon_{n p}^{(1)}-\mu}{k_{\mathrm{B}} T} \exp \frac{\varepsilon_{n / p^{\prime}}^{(2)}-\mu}{k_{\mathrm{B}} T} f\left(\varepsilon_{n p}^{(1)}-\mu\right) f\left(\varepsilon_{n^{\prime} p^{\prime}}^{(2)}-\mu-\frac{e V}{2}\right) f\left(\varepsilon_{n p^{\prime}}^{(1)}-\mu\right) f\left(\varepsilon_{n^{\prime} p}^{(2)}-\mu+\frac{e V}{2}\right)
$$

$\kappa$ is the dielectric susceptibility of the sample and

$$
g_{n n^{\prime}}(q)=\left[\int d \mathbf{r}_{\perp} \int d \mathbf{r}_{\perp}^{\prime}\left|\phi_{n}\left(\mathbf{r}_{\perp}\right)\right|^{2} K_{0}\left(\left|q \| \mathbf{r}_{\perp}-\mathbf{r}_{\perp}^{\prime}\right| / \hbar\right)\left|\phi_{n^{\prime}}\left(\mathbf{r}_{\perp}^{\prime}\right)\right|^{2}\right]^{2} .
$$

Some estimates of this function are given in Ref. [1].

According to the reasoning given in Ref. [1] all the terms of the sum (13) where the differences $\left|\varepsilon^{(1)}(0)-\varepsilon^{(2)}(0)\right|$ are much bigger than both $k_{\mathrm{B}} T$ and $e V$ do not contribute to the current $J$. Therefore we are left with the terms of the sum where $\left|\varepsilon^{(1)}(0)-\varepsilon^{(2)}(0)\right|$ is smaller 
than or of the order of $k_{\mathrm{B}} T$ or $e V$. We will assume for simplicity that there is only one such difference (otherwise we would have gotten a sum of several terms of the same structure).

As $\mathcal{Q}$ is a sharp function of $p$ and $p^{\prime}$ one can take out of the integral all the slowly varying functions and get

$$
J=J_{0} \cdot \frac{1}{2} \sinh \left(\frac{e V}{2 k_{\mathrm{B}} T}\right) \frac{\frac{e V}{4 k_{\mathrm{B}} T}-\frac{\varepsilon_{n n^{\prime}}}{2 k_{\mathrm{B}} T}}{\sinh \left(\frac{e V}{4 k_{\mathrm{B}} T}-\frac{\varepsilon_{n n^{\prime}}}{2 k_{\mathrm{B}} T}\right)} \cdot \frac{\frac{e V}{4 k_{\mathrm{B}} T}+\frac{\varepsilon_{n n^{\prime}}}{2 k_{\mathrm{B}} T}}{\sinh \left(\frac{e V}{4 k_{\mathrm{B}} T}+\frac{\varepsilon_{n n^{\prime}}}{2 k_{\mathrm{B}} T}\right)}
$$

where

$$
J_{0}=-\frac{8 e^{5} m^{3} L\left(k_{\mathrm{B}} T\right)^{2}}{\kappa^{2} \pi^{2} \hbar^{4}} \cdot \frac{g_{n n^{\prime}}\left(2 p_{n}\right)}{p_{n}^{3}}
$$

Here we have introduced notation

$$
\varepsilon_{n n^{\prime}}=\varepsilon_{n}^{(1)}(0)-\varepsilon_{n^{\prime}}^{(2)}(0), \quad m v_{n}=p_{n}=\sqrt{2 m\left[\mu-\varepsilon_{n}^{(1)}(0)\right]}
$$

Let us give an order-of-magnitude estimate of the drag current $J$ for a realistic situation. We assume $T=1 \mathrm{~K}, \mu=14 \mathrm{meV}$, the widths of the wires are $25 \mathrm{~nm}$, the distance between thr central lines of the wires is $50 \mathrm{~nm}, \kappa=13, m=6.7 \cdot 10^{-29}$. Then

$$
J_{0} \approx 10^{-10} \mathrm{~A}
$$

For $e V \ll k_{\mathrm{B}} T$ one gets from Eq. (16) the result of Ref. [1]. Let us consider in detail the opposite case $e V \gg k_{\mathrm{B}} T$. In this case one gets a nonvanishing result for Eq.(16) only if $\left|\varepsilon_{n n \prime}\right|<e V / 2$ and one obtains the following equation for the drag current

$$
J=\mathcal{B}\left[\left(\frac{e V}{2}\right)^{2}-\left(\varepsilon_{n n^{\prime}}\right)^{2}\right], \quad \mathcal{B}=-\frac{2 e^{5} m^{3} L}{\kappa^{2} \pi^{2} \hbar^{4}} \cdot \frac{g_{n n^{\prime}}\left(2 p_{n}\right)}{p_{n}^{3}} .
$$

The situation is illustrated in Fig. 1. The straight lines correspond to the positions of the chemical potentials $\mu^{(r)}$ and $\mu^{(l)}$ while the dashed line corresponds to the average value $\mu$. Parabolas (1) and (2) represent the dispersion law of electrons in wires (1) and (2) respectively. The full circles correspond to the initial states of colliding electrons.

Before the collision states $1 \mathrm{a}$ and $2 \mathrm{a}$ are occupied. The circle representing state 1a is below the dashed line, i.e. below the Fermi level $\mu$. The circle 2 a represents a state with $p>0$ which is also occupied as the corresponding energy is below $\mu^{(r)}$. 
After the collision state $1 \mathrm{~b}$ is occupied. It is represented by a circle above the dashed line which means that it has been free before the collision. In wire 2 state $2 \mathrm{~b}$ with $p<0$ is also occupied. It is above $\mu^{(l)}$, i.e. it has been free before the transition.

The width of the stripe between the two straight lines is $e V$. The drag current should be proportional to the number of the occupied initial states as well as to the number of free final states. As a result we have for $e V \gg\left|\varepsilon_{n n^{\prime}}\right|, \quad J \propto V^{2}$ - see Fig. 2.

In summary, we have developed a theory of Coulomb drag current in a quantum wire brought about by a non-Ohmic current in a nearby parallel nanowire. A ballistic transport in both nanowires is assumed. The drag current $J$ as a function of the gate voltage comprises a system of spikes; the position of each spike is determined by a coincidence of a pair of levels of transverse quantization, $\varepsilon_{n}(0)$ and $\varepsilon_{n^{\prime}}(0)$ in both wires. For $e V \gg k_{\mathrm{B}} T, J$ is a parabolic function of the driving voltage $V$. The effect may play an important role in the investigation of the interwire Coulomb scattering as well as 1D band structure of the wires.

The authors are grateful to P. Debray for sending them a preprint of paper [2] prior to publication. The authors are pleased to acknowledge the support for this work by the Russian National Fund of Fundamental Research (Grant No 97-02-18286-a). 


\section{REFERENCES}

[1] V. L. Gurevich, V. B. Pevzner, and E. W. Fenton, J. Phys.: Condens. Matter 10, 2551, (1998)

[2] P. Debray, P. Vasilopulos, O. Raichev, R. Perrin, M. Rahman and W. C. Mitchel, preprint.

[3] R. Landauer, IBM J. Res. Develop. 1, 233 (1957); 32(3), 306 (1989).

[4] Y. Imry, Directions in Condensed Matter Physics, ed. G. Grinstein and G. Mazenko, 1986 (Singapore: World Scientific), 101; M. Büttiker, Phys. Rev. Lett., 57, 1761 (1986).

\section{FIGURE CAPTIONS}

Fig. 1. Schematic representation of simultaneous transitions due to the interaction between electrons of the two wires for $\mathrm{eV} \gg k_{\mathrm{B}} T$. Circles $\circ$ and $\bullet$ represent the initially unoccupied and occupied states respectively.

Fig. 2. Dependence of the drag current on the driving voltage for $T=1 \mathrm{~K}$. The values of $\left|\varepsilon_{n n^{\prime}}\right|$ go up from left to right. 